\title{
Needles galore, but no place to sew
}

\section{Large-scale sequencing projects to identify mutations related to cancer and psychiatric disorders will generate a lot of data. But the usefulness of these results will be limited unless we have good models to test their contribution to disease.}

$\mathbf{F}$ irst, it was the Human Genome Project. Although it had its fair share of critics, there was consensus that sequencing the whole human genome-the blueprint of life, as some called it—was a good idea. Then, the Cancer Genome Atlas came along, and critics wondered whether it made sense to spend US $\$ 1$ billion to create a catalog of all the mutations found in human cancers, particularly in a climate of less money for investigatordriven research. Now, a new large-scale sequencing project aimed at uncovering the genes relevant to psychiatric illnesses has come to the fore. This project is privately funded-the Stanley Medical Research Institute has awarded US \$100 million to the Broad Institute to carry it out-but questions remain about the returns that can be expected on this hefty investment.

There is no question that both projects will yield vast amounts of data. In fact, several recent papers already show how prolific this approach will be. In one of them (Nature 446, 153-158; 2007), the authors identified 1,007 mutations in 518 protein kinase genes from 210 human cancers, ultimately proposing that 120 of these genes carry 'driver' mutations-mutations that might be causally implicated in tumor development. These results are striking in terms of their sheer volume, but how much closer do they bring us to understanding cancer?

In the drug-discovery world, the signal-to-noise ratio is a key concern during high-throughput screening of a chemical library. You want to get enough 'hits' for further analysis, but if you get too many, then there's something wrong with the sensitivity of your screening assay. If we apply this principle to the results of the Nature paper, the authors got a staggering hit rate of over $20 \%$. Moreover, related studies have identified other sets of driver genes. Do more than $20 \%$ of the sequenced genes actually carry driver mutations, or is the usefulness of the approach limited by a very low signal-to-noise ratio? It is indeed theoretically possible that the number of cancer genes is very large. But if one in five kinases that we choose to study gives us a hit, then one wonders about the benefits of spending $\$ 1$ billion to search in a haystack that seems to have needles galore.

In all fairness, researchers are well aware of the limitations of large-scale sequencing projects and refer to the genes identified as 'candidate' genes. As both critics and advocates of the screening approach agree, we won't know if a specific gene is causally linked to disease until we perform studies to analyze its function. It is therefore unfortunate that we often lack good models to test the pathophysiological relevance of this plethora of candidates. So, even if the sequencing efforts were doing a great job at revealing strong candidate genes, we don't always have the tools to nail down their true contribution to disease.

We have good animal models of some cancers, but there are types for which the existing models remain somewhat inadequate to rigorously test the relevance of a gene to pathology. In the case of psychiatric disorders, the inadequacy of the models is even more apparent. Strategies such as paired-pulse inhibition (a model of schizophrenia), learned helplessness (a model of depression) and the elevated T-maze (a model of anxiety) are widely regarded as too simplistic to accurately mirror the human disease, and will be of limited use in helping us determine if new candidate genes are causally linked to mental illness. If we now consider that psychiatric diseases are polygenic (many genes are relevant) and that estimates of the heritability of conditions such as depression are low (the contributions of each of those genes to disease is very small), then the situation is rather grim: limited genetic contribution, many candidate genes and no good models to test their functional role.

Scientists may disagree on how useful they think large-scale sequencing projects will ultimately be. But the reality is that these projects are pushing forward and, now that results are beginning to surface, it is in everyone's interest to think of new ways to take advantage of the outcome of these ventures.

Signal-to-noise ratio is a cause for concern, as is the lack of models to test the functional relevance of candidate genes. And although the first criticism is aimed directly at the sequencing efforts, the second one is relevant to the whole biomedical community, which has not paid enough attention to developing reliable models of disease. It is therefore crucial for funding bodies to be as generous with projects aimed at developing new models as they have been with the sequencing endeavors. Otherwise, we'll end up with too many needles but very little fabric. 